Research Article

\title{
Synchronization of Two Rank-One Chaotic Systems without and with Delay via Linear Delayed Feedback Control
}

\author{
Hui Fang \\ Department of Mathematics, Kunming University of Science and Technology, Yunnan 650093, China \\ Correspondence should be addressed to Hui Fang, kmustfanghui@hotmail.com \\ Received 22 November 2011; Accepted 4 January 2012 \\ Academic Editor: Wan-Tong Li \\ Copyright (C) 2012 Hui Fang. This is an open access article distributed under the Creative \\ Commons Attribution License, which permits unrestricted use, distribution, and reproduction in \\ any medium, provided the original work is properly cited. \\ This paper illustrates the presence of chaos in rank-one chaotic systems with delay via a binary test \\ (called 0-1 test) for chaos. Chaotic synchronization between two rank-one chaotic systems without \\ and with delay is achieved by means of Lyapunov functional and linear delayed feedback control \\ method. Numerical simulations are implemented to verify the effectiveness of the proposed chaos \\ synchronization scheme.
}

\section{Introduction}

The introduction of the notion of chaotic synchronization by Pecora and Carroll [1] has stimulated a wide range of research activity in various fields. In 1999, Mainieri and Rehacek proposed a new method of synchronization, that is, projective synchronization [2]. Since then, many papers have been published on the projective synchronization of chaotic systems (see [3-14] and references cited therein). For some recent works on the synchronization of delayed chaotic systems, we refer the reader to [14-16].

Wang and Young first developed a general theory of rank-one attractors, that is, attractors with a single direction of instability and strong contraction in all other directions, then applied it to prove the existence of rank-one attractors in certain systems of differential equations [17-19]. In 2005, Wang and Oksasoglu presented a new mechanism of producing rank-one chaos in Chua's system [20]. In 2009, Chen and Han studied the existence of rankone chaos in the neighborhood of a periodically kicked stable limit cycle close to a heteroclinic cycle of a planar equation [21]. However, to our knowledge, few papers have been published on the synchronization of rank-one chaotic systems. 
In this paper, we study the synchronization between rank-one chaotic systems without and with delay using linear control method. The detailed arrangement is as follows: in Section 2, the rank-one chaotic systems proposed in $[20,21]$ are briefly described. The presence of chaos in rank-one chaotic systems with delay are tested using the $0-1$ test for chaos recently proposed in [22]. In Section 3, the linear delayed feedback control method is adopted to realize the synchronization between two rank-one chaotic systems without and with delay. In Section 4, numerical simulations are shown to verify the validity and feasibility of the proposed method, and finally some conclusions are given in Section 5.

\section{Systems Description}

Let

$$
P_{T, p}(t)=\frac{1}{p} \sum_{n=-\infty}^{+\infty} F_{n, T, p}(t)
$$

where

$$
F_{n, T, p}(t)= \begin{cases}1, & n T \leq t<n T+p \\ 0, & \text { elsewhere }\end{cases}
$$

$P_{T, p}(t)$ is a periodic pulse train with a pulse width of $p$ and a period of $T$.

The rank-one chaotic system proposed in [20] can be described as follows:

$$
\begin{gathered}
\dot{x}=\alpha\left[y-\beta_{1} x-\beta_{3} x^{3}\right]+\eta x P_{T, p}(t), \\
\dot{y}=x-y+z \\
\dot{z}=-\beta y
\end{gathered}
$$

where $\eta$ is used for controlling the magnitude of the forcing. When the system parameters are taken as the following values:

$$
\alpha=2, \quad \beta=2.31135, \quad \beta_{1}=0.2495, \quad \beta_{3}=-1, \quad \eta=0.15, \quad p=0.5,
$$

there exist rank-one attractors in system (2.3) for large enough $T$, such as $T=95$ [20].

The rank-one chaotic system proposed in [21] can be described as follows:

$$
\begin{gathered}
\dot{x}=2 B x y+\varepsilon\left(x+a x^{2}\right)-\varepsilon_{1} x(t) P_{T, p}(t), \\
\dot{y}=1-2 A x-B y^{2},
\end{gathered}
$$

where $\varepsilon_{1}$ is used to control the magnitude of the forcing. When $A=1, B=1, \varepsilon=-1, a=-1.23$, $p=0.5, \varepsilon_{1}=0.4$, there exist rank-one attractors in system (2.5) for $T=10$ and $T=8$ [21]. 
Without the particular statement, the above parameter values are adopted in this whole paper.

Incorporating delays into (2.3), (2.5) due to the effect of propagation delay in practical circuits, we consider the following systems:

$$
\begin{gathered}
\dot{x}=\alpha\left[y(t-\tau)-\beta_{1} x(t)-\beta_{3} x^{3}(t)\right]+\eta x(t) P_{T, p}(t), \\
\dot{y}=x(t-\tau)-y(t)+z(t-\tau), \\
\dot{z}=-\beta y(t-\tau) ; \\
\dot{x}=2 B x(t) y(t)+\varepsilon\left[x(t)+a x^{2}(t)\right]-\varepsilon_{1} x(t) P_{T, p}(t), \\
\dot{y}=1-2 A x(t-\tau)-B y^{2}(t) .
\end{gathered}
$$

The presence of chaos in systems (2.6), (2.7) will be confirmed via the $0-1$ test for chaos. For convenience, we briefly describe the test as follows. For more details, we refer the reader to [22-24].

Consider a set of discrete data $\phi(n)(n=1,2,3, \ldots)$, representing a one-dimensional observable dataset obtained from the underlying dynamics, such as a set of experimental data or a set of discrete data obtained from numerical solutions. For $c \in(0, \pi)$, define the translation variables as follows:

$$
p_{c}(n)=\sum_{j=1}^{n} \phi(j) \cos j c, \quad q_{c}(n)=\sum_{j=1}^{n} \phi(j) \sin j c, \quad n=1,2,3, \ldots, N .
$$

To determine the growth of $p_{c}$ and $q_{c}$, it is convenient to look at the mean square displacement (MSD), defined as

$$
M_{c}(n)=\lim _{N \rightarrow \infty} \frac{1}{N} \sum_{j=1}^{N}\left[\left(p_{c}(j+n)-p_{c}(j)\right)^{2}+\left(q_{c}(j+n)-q_{c}(j)\right)^{2}\right] .
$$

The test for chaos is based on the asymptotic growth rate $k_{c}$ of $M_{c}(n)$ as a function of $n$, which can be estimated as follows:

$$
k_{c}=\lim _{n \rightarrow \infty} \frac{\log M_{c}(n)}{\log n} .
$$

Numerically, $k_{c}$ is determined by fitting a straight line to the graph of $\log M_{c}(n)$ versus $\log n$ through minimizing the absolute deviation.

For different $c$ values, different $k_{c}$ values can be calculated. The underlying dynamics should be examined by whether the median $k$ of $k_{c}$ values approach 0 or 1 . Specifically, $k$ is close to 0 , signifying nonchaotic dynamics (i.e., periodic or quasi-periodic), or $k$ is close to 1 , signifying chaotic dynamics. Moreover, the dynamics of the translation components $(p, q)$ are corresponding to the underlying dynamics. Bounded trajectories are corresponding 
to periodic or quasi-periodic dynamics and Brownian-like (unbounded) trajectories are corresponding to chaotic dynamics.

For a sampled time series $x(t)$ for system (2.6), we have the following results:

(1) $k=0.9980$ for $\tau=0.001, k=0.9974$ for $\tau=0.003, k=0.9978$ for $\tau=0.01$, indicating that the dynamics of system (2.6) are chaotic. (a), (b), and (c) in Figure 1 depict Brownian-like trajectories, confirming that the dynamics of system (2.6) are chaotic for $\tau=0.001, \tau=0.003, \tau=0.01$;

(2) $k=0.0561$ for $\tau=0.06$, indicating that the dynamics of system (2.6) are nonchaotic. (d) in Figure 1 depicts a bounded trajectory, confirming that the dynamics of system (2.6) is nonchaotic for $\tau=0.06$.

For a sampled time series $x(t)$ for system (2.7), we have the following results: $k=$ 0.9977 for $\tau=0.03, T=8$, and $k=0.9978$ for $\tau=0.03, T=10$, indicating that the dynamics of system (2.7) are chaotic. (a) and (b) in Figure 2 depict Brownian-like trajectories, confirming that the dynamics of system (2.7) are chaotic for $T=8, T=10$, and $\tau=0.03$.

\section{Synchronization between Two Rank-one Chaotic Systems without and with Delay}

The aim of this section is to achieve chaos synchronization for two rank-one chaotic systems without and with delay using linear delayed feedback control method.

We define the drive and response systems as follows:

$$
\begin{gathered}
\dot{X}_{d}=F\left(t, X_{d}(t)\right), \\
\dot{X}_{r}=G\left(t, X_{r}(t), X_{r}(t-\tau)\right)+U(t),
\end{gathered}
$$

where $X_{d}(t)=\left[X_{d}^{1}(t), \ldots, X_{d}^{m}(t)\right]^{T} \in R^{m}, X_{r}(t)=\left[X_{r}^{1}(t), \ldots, X_{r}^{n}(t)\right]^{T} \in R^{n}, \tau \geq 0$, and $U(t)=$ $\left[u_{1}(t), \ldots, u_{n}(t)\right]^{T}$ is a controller and that can be called the control function.

For brevity, let us define the synchronous error as follows:

$$
\left[e_{1}(t), \ldots, e_{n}(t)\right]^{T}=X_{r}(t)-K_{n \times m} X_{d}(t),
$$

where $K_{n \times m}$ is a constant scaling matrix.

Before presenting the main results, we introduce the following Barbalat's lemma and definition.

Lemma 3.1 (Barbalat's Lemma, [25, Lemma 1.2.2, page 4]). Let $f$ be a nonnegative function defined on $[0,+\infty)$ such that $f$ is integrable on $[0,+\infty)$ and is uniformly continuous on $[0,+\infty)$. Then, $\lim _{t \rightarrow+\infty} f(t)=0$.

Definition 3.2 (See [2]). The drive system (3.1) and the response system (3.2) are said to realize projective synchronization, if there exists a tracking control $U(t)=\left[u_{1}(t), \ldots, u_{n}(t)\right]^{T}$ such that the synchronous errors satisfy

$$
\lim _{t \rightarrow+\infty} e_{i}(t)=0 \quad(i=1,2, \ldots, n) .
$$




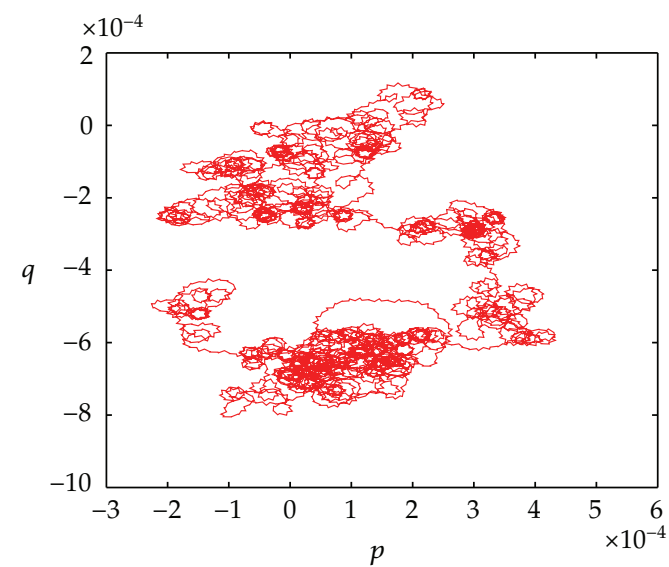

(a)

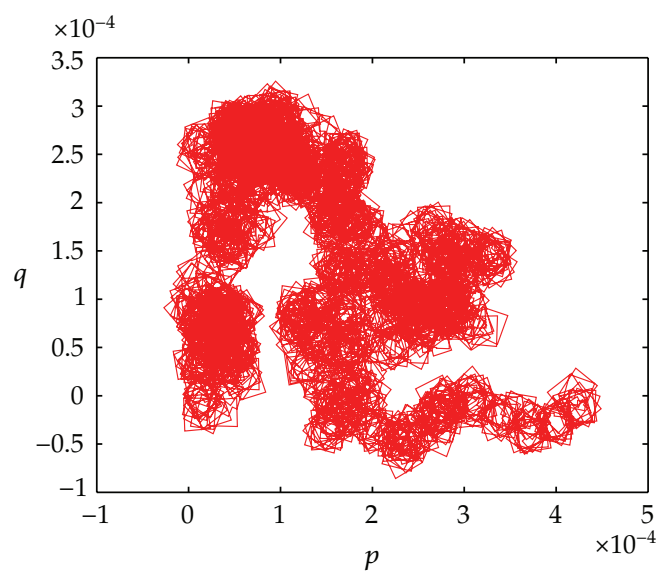

(c)

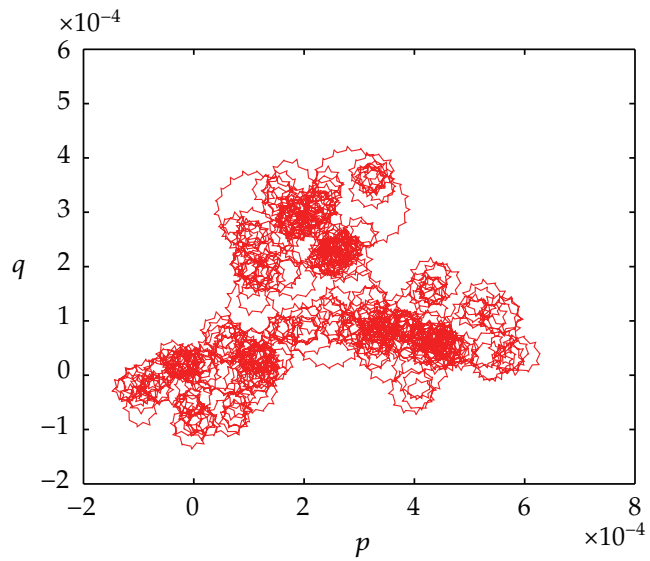

(b)

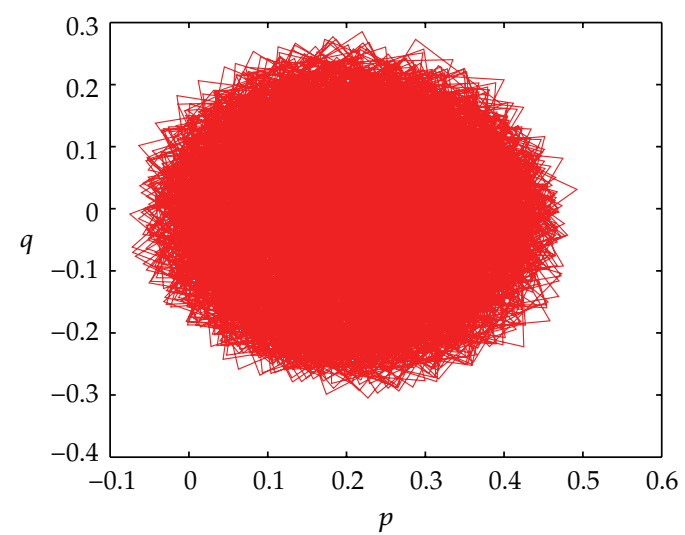

(d)

Figure 1: Dynamics of the translation components $(p, q)$ of system (2.6): (a), (b), and (c) show Brownianlike trajectories for $\tau=0.001, \tau=0.003, \tau=0.01$, respectively; (d) shows a bounded trajectory for $\tau=0.06$.

Consider the drive system (3.5) and the response system (3.6):

$$
\begin{gathered}
\dot{x}_{1}=\alpha\left[y_{1}(t)-\beta_{1} x_{1}(t)-\beta_{3} x_{1}^{3}(t)\right]+\eta x_{1}(t) P_{T, p}(t), \\
\dot{y}_{1}=x_{1}(t)-y_{1}(t)+z_{1}(t), \\
\dot{z}_{1}=-\beta y_{1}(t), \\
\dot{x}_{2}=\alpha\left[y_{2}(t-\tau)-\beta_{1} x_{2}(t)-\beta_{3} x_{2}^{3}(t)\right]+\eta x_{2}(t) P_{T, p}(t)+u_{1}(t), \\
\dot{y}_{2}=x_{2}(t-\tau)-y_{2}(t)+z_{2}(t-\tau)+u_{2}(t), \\
\dot{z}_{2}=-\beta y_{2}(t-\tau)+u_{3}(t),
\end{gathered}
$$

where $u(t)=\left[u_{1}(t), u_{2}(t), u_{3}(t)\right]^{T}$ is the control function. 


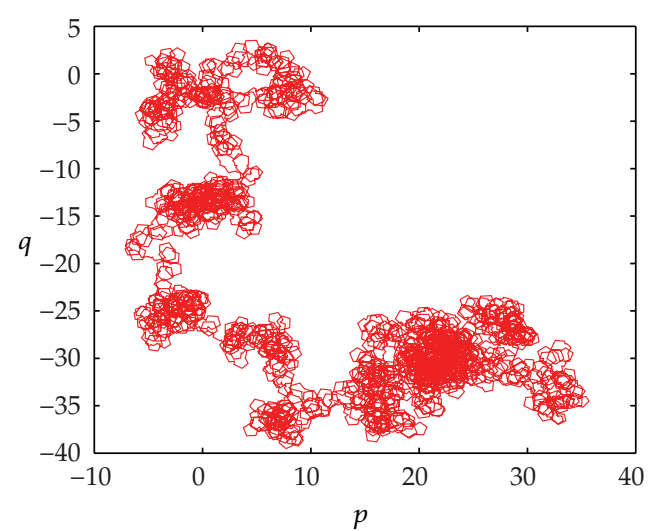

(a)

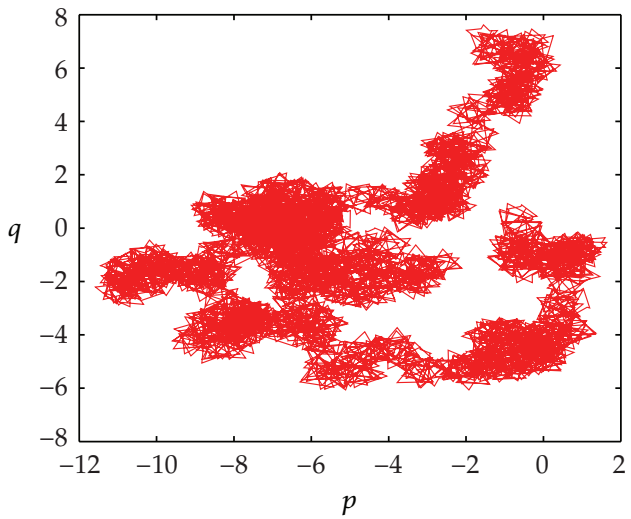

(b)

Figure 2: Dynamics of the translation components $(p, q)$ of system (2.7) with $\tau=0.03$ : (a) and (b) show Brownian-like trajectories for $T=8, T=10$, respectively.

In this case, we define the synchronous errors as follows:

$$
\begin{aligned}
& e_{1}(t)=x_{2}(t)-k_{1} x_{1}(t) \\
& e_{2}(t)=y_{2}(t)-k_{2} y_{1}(t) \\
& e_{3}(t)=z_{2}(t)-k_{3} z_{1}(t)
\end{aligned}
$$

Now, we present the main result for the synchronization between system (3.5) and system (3.6).

Theorem 3.3. If the linear controller $u(t)=\left[u_{1}(t), u_{2}(t), u_{3}(t)\right]^{T}$ is given as follows:

$$
\begin{gathered}
u_{1}(t)=\alpha\left[k_{1} y_{1}(t)-k_{2} y_{1}(t-\tau)\right]-m_{1}\left[x_{2}(t)-k_{1} x_{1}(t)\right], \\
u_{2}(t)=k_{2} x_{1}(t)-k_{1} x_{1}(t-\tau)+k_{2} z_{1}(t)-k_{3} z_{1}(t-\tau)-m_{2}\left[y_{2}(t)-k_{2} y_{1}(t)\right], \\
u_{3}(t)=\beta\left[k_{2} y_{1}(t-\tau)-k_{3} y_{1}(t)\right]-m_{3}\left[z_{2}(t)-k_{3} z_{1}(t)\right],
\end{gathered}
$$

where $k_{1}= \pm 1, m_{1}>1+\eta / p-\alpha \beta_{1}-0.1875 \alpha \beta_{3}, m_{2}>\alpha+\beta-1, m_{3}>1$. Then, the drive system (3.5) and the response system (3.6) realize projective synchronization.

Proof. Because $\left|x_{1}(t)\right|<0.25$ for sufficiently large $t \geq t_{0}^{*}$ [20], we make the following prior assumption:

$$
\left|x_{2}(t)\right|<0.25, \quad t \geq t_{0}\left(t_{0} \geq t_{0}^{*}\right)
$$

Consider the Lyapunov functional defined by

$$
V(t)=\left|e_{1}(t)\right|+\left|e_{2}(t)\right|+\left|e_{3}(t)\right|+\int_{t-\tau}^{t}\left[\left|e_{1}(s)\right|+(\alpha+\beta)\left|e_{2}(s)\right|+\left|e_{3}(s)\right|\right] d s, \quad t \geq t_{0} .
$$


Calculating the upper right Dini derivative $D^{+} V(t)$ of $V(t)$ along the solutions of system (3.5) and system (3.6), we get

$$
\begin{aligned}
D^{+} V(t) \leq & \tilde{D}_{1}(t)+\tilde{D}_{2}(t)+\widetilde{D}_{3}(t)+\left|e_{1}(t)\right|-\left|e_{1}(t-\tau)\right| \\
& +(\alpha+\beta)\left[\left|e_{2}(t)\right|-\left|e_{2}(t-\tau)\right|\right]+\left|e_{3}(t)\right|-\left|e_{3}(t-\tau)\right|
\end{aligned}
$$

where

$$
\widetilde{D}_{i}(t)=D^{+}\left|e_{i}(t)\right|= \begin{cases}\dot{e}_{i}(t), & e_{i}(t)>0 \\ -\dot{e}_{i}(t), & e_{i}(t)<0, \quad(i=1,2,3) \\ \left|\dot{e}_{i}(t)\right|, & e_{i}(t)=0\end{cases}
$$

There are the following three cases to consider for $\tilde{D}_{1}(t)$.

(i) If $e_{1}(t)>0$ (i.e., $\left.x_{2}(t)>k_{1} x_{1}(t)\right)$, then

$$
\begin{aligned}
\tilde{D}_{1}(t) & =-\left(\alpha \beta_{1}+m_{1}\right) e_{1}(t)+\alpha e_{2}(t-\tau)-\alpha \beta_{3}\left[x_{2}^{3}(t)-k_{1} x_{1}^{3}(t)\right]+\eta e_{1}(t) P_{T, p}(t) \\
& \leq-\left(\alpha \beta_{1}-\frac{\eta}{p}+m_{1}\right)\left|e_{1}(t)\right|+\alpha\left|e_{2}(t-\tau)\right|-\alpha \beta_{3}\left|e_{1}(t)\right|\left|x_{2}^{2}(t)+k_{1} x_{1}(t) x_{2}(t)+k_{1}^{2} x_{1}^{2}(t)\right| \\
& \leq-\left[\alpha \beta_{1}-\frac{\eta}{p}+0.1875 \alpha \beta_{3}+m_{1}\right]\left|e_{1}(t)\right|+\alpha\left|e_{2}(t-\tau)\right| .
\end{aligned}
$$

(ii) If $e_{1}(t)<0$ (i.e., $x_{2}(t)<k_{1} x_{1}(t)$ ), then

$$
\begin{aligned}
\tilde{D}_{1}(t) & =\left(\alpha \beta_{1}+m_{1}\right) e_{1}(t)-\alpha e_{2}(t-\tau)+\alpha \beta_{3}\left[x_{2}^{3}(t)-k_{1}^{3} x_{1}^{3}(t)\right]-\eta e_{1}(t) P_{T, p}(t) \\
& \leq-\left[\alpha \beta_{1}-\frac{\eta}{p}+0.1875 \alpha \beta_{3}+m_{1}\right]\left|e_{1}(t)\right|+\alpha\left|e_{2}(t-\tau)\right|
\end{aligned}
$$

(iii) If $e_{1}(t)=0$ (i.e., $\left.x_{2}(t)=k_{1} x_{1}(t)\right)$, then

$$
\widetilde{D}_{1}(t)=\alpha\left|e_{2}(t-\tau)\right|=-\left[\alpha \beta_{1}-\frac{\eta}{p}+0.1875 \alpha \beta_{3}+m_{1}\right]\left|e_{1}(t)\right|+\alpha\left|e_{2}(t-\tau)\right| .
$$

From (i)-(iii), we have

$$
\tilde{D}_{1}(t) \leq-\left[\alpha \beta_{1}-\frac{\eta}{p}+0.1875 \alpha \beta_{3}+m_{1}\right]\left|e_{1}(t)\right|+\alpha\left|e_{2}(t-\tau)\right|, \quad t \geq t_{0}
$$


It is easy to see that

$$
\begin{gathered}
\tilde{D}_{2}(t)= \begin{cases}e_{1}(t-\tau)-\left(1+m_{2}\right) e_{2}(t)+e_{3}(t-\tau), & e_{2}(t)>0, \\
-e_{1}(t-\tau)+\left(1+m_{2}\right) e_{2}(t)-e_{3}(t-\tau), & e_{2}(t)<0, \\
\left|e_{1}(t-\tau)+e_{3}(t-\tau)\right|, & e_{2}(t)=0,\end{cases} \\
\tilde{D}_{3}(t)= \begin{cases}-\beta e_{2}(t-\tau)-m_{3} e_{3}(t), & e_{3}(t)>0, \\
\beta e_{2}(t-\tau)+m_{3} e_{3}(t), & e_{3}(t)<0, \\
\beta\left|e_{2}(t-\tau)\right|, & e_{3}(t)=0 .\end{cases}
\end{gathered}
$$

Considering $\widetilde{D}_{2}(t), \widetilde{D}_{3}(t)$ in the same way, we can obtain

$$
\begin{gathered}
\tilde{D}_{2}(t) \leq-\left(1+m_{2}\right)\left|e_{2}(t)\right|+\left|e_{1}(t-\tau)\right|+\left|e_{3}(t-\tau)\right|, \quad t \geq t_{0} \\
\tilde{D}_{3}(t) \leq-m_{3}\left|e_{3}(t)\right|+\beta\left|e_{2}(t-\tau)\right|, \quad t \geq t_{0} .
\end{gathered}
$$

Hence, we have

$$
\begin{aligned}
D^{+} V(t) \leq & \widetilde{D}_{1}(t)+\widetilde{D}_{2}(t)+\widetilde{D}_{3}(t)+\left|e_{1}(t)\right|-\left|e_{1}(t-\tau)\right| \\
& +(\alpha+\beta)\left[\left|e_{2}(t)\right|-\left|e_{2}(t-\tau)\right|\right]+\left|e_{3}(t)\right|-\left|e_{3}(t-\tau)\right| \\
\leq & -\left[\alpha \beta_{1}-\frac{\eta}{p}+0.1875 \alpha \beta_{3}+m_{1}-1\right]\left|e_{1}(t)\right|-\left(1+m_{2}-\alpha-\beta\right)\left|e_{2}(t)\right| \\
& -\left(m_{3}-1\right)\left|e_{3}(t)\right|, \quad t \geq t_{0} .
\end{aligned}
$$

So there exists $c>0$ such that

$$
D^{+} V(t) \leq-c\left(\left|e_{1}(t)\right|+\left|e_{2}(t)\right|+\left|e_{3}(t)\right|\right), \quad t \geq t_{0}
$$

Integrating on both sides of the above inequality leads to

$$
V(t)+c \int_{t_{0}}^{t}\left[\left|e_{1}(s)\right|+\left|e_{2}(s)\right|+\left|e_{3}(s)\right|\right] d s \leq V\left(t_{0}\right)<+\infty, \quad t \geq t_{0},
$$

which implies

$$
\left|e_{1}(t)\right|+\left|e_{2}(t)\right|+\left|e_{3}(t)\right| \in L^{1}[0,+\infty) .
$$


From the boundedness of $x_{i}(t), y_{i}(t)$, and $z_{i}(t)(i=1,2)$, it follows that $e_{1}(t), e_{2}(t), e_{3}(t)$, and their derivatives remain bounded on $[0,+\infty)$. Hence, $\left|e_{1}(t)\right|+\left|e_{2}(t)\right|+\left|e_{3}(t)\right|$ is uniformly continuous. By Lemma 3.1, it follows that

$$
\lim _{t \rightarrow+\infty}\left(\left|e_{1}(t)\right|+\left|e_{2}(t)\right|+\left|e_{3}(t)\right|\right)=0 .
$$

Hence,

$$
\lim _{t \rightarrow+\infty}\left|e_{1}(t)\right|=0, \quad \lim _{t \rightarrow+\infty}\left|e_{2}(t)\right|=0, \quad \lim _{t \rightarrow+\infty}\left|e_{3}(t)\right|=0 .
$$

The proof is complete.

Remark 3.4. When $k_{1}=k_{2}=k_{3}=1, \tau=0$, the linear controller $u(t)=\left[u_{1}(t), u_{2}(t), u_{3}(t)\right]^{T}$ is given as follows:

$$
u_{1}(t)=-m_{1}\left[x_{2}(t)-x_{1}(t)\right], \quad u_{2}(t)=-m_{2}\left[y_{2}(t)-y_{1}(t)\right], \quad u_{3}(t)=-m_{3}\left[z_{2}(t)-z_{1}(t)\right] .
$$

In this case, the drive system (3.5) and the response system (3.6) realize complete synchronization.

Remark 3.5. When $k_{1}=k_{2}=k_{3}=-1, \tau=0$, the linear controller $u(t)=\left[u_{1}(t), u_{2}(t), u_{3}(t)\right]^{T}$ is given as follows:

$$
u_{1}(t)=-m_{1}\left[x_{2}(t)+x_{1}(t)\right], \quad u_{2}(t)=-m_{2}\left[y_{2}(t)+y_{1}(t)\right], \quad u_{3}(t)=-m_{3}\left[z_{2}(t)+z_{1}(t)\right] .
$$

In this case, the drive system (3.5) and the response system (3.6) realize antisynchronization.

Consider the drive system (3.27) and the response system (3.28):

$$
\begin{gathered}
\dot{x}_{1}=2 B x_{1}(t) y_{1}(t)+\varepsilon\left[x_{1}(t)+a x_{1}^{2}(t)\right]-\varepsilon_{1} x_{1}(t) P_{T, p}(t), \\
\dot{y}_{1}=1-2 A x_{1}(t)-B y_{1}^{2}(t), \\
\dot{x}_{2}=2 B x_{2}(t) y_{2}(t)+\varepsilon\left[x_{2}(t)+a x_{2}^{2}(t)\right]-\varepsilon_{1} x_{2}(t) P_{T, p}(t)+u_{1}(t), \\
\dot{y}_{2}=1-2 A x_{2}(t-\tau)-B y_{2}^{2}(t)+u_{2}(t),
\end{gathered}
$$

where $u=\left[u_{1}(t), u_{2}(t)\right]^{T}$ is the control function.

Our goal is to determine the control function $u=\left[u_{1}(t), u_{2}(t)\right]^{T}$. For brevity, let us define

$$
e_{1}(t)=x_{2}(t)-x_{1}(t), \quad e_{2}(t)=y_{2}(t)-y_{1}(t)
$$


Now, we present the main result for the synchronization between system (3.27) and system (3.28).

Theorem 3.6. If the linear controller $u=\left[u_{1}(t), u_{2}(t)\right]^{T}$ is given as follows:

$$
\begin{gathered}
u_{1}(t)=-m_{1}\left[x_{2}(t)-x_{1}(t)\right] \\
u_{2}(t)=2 A\left[x_{1}(t-\tau)-x_{1}(t)\right]-m_{2}\left[y_{2}(t)-y_{1}(t)\right],
\end{gathered}
$$

where $m_{1}>2 A+\varepsilon a, m_{2}>4 B$. Then, $e_{1}, e_{2}$ converge to zero as time $t$ tends to infinity, that is, the drive system (3.27) and the response system (3.28) asymptotically synchronize.

Proof. Because $0<x_{1}(t)<1,\left|y_{1}(t)\right|<1$ for sufficiently large $t \geq t_{0}^{*}[21]$, we make the following prior assumption:

$$
0<x_{2}(t)<1, \quad\left|y_{2}(t)\right|<1, \quad t \geq t_{0}\left(t_{0} \geq t_{0}^{*}\right)
$$

Consider the Lyapunov functional defined by

$$
V(t)=\left|\ln x_{2}(t)-\ln x_{1}(t)\right|+\left|y_{2}(t)-y_{1}(t)\right|+2 A \int_{t-\tau}^{t}\left|x_{2}(s)-x_{1}(s)\right| d s, \quad t \geq t_{0} .
$$

Calculating the upper right Dini derivative $D^{+} V(t)$ of $V(t)$ along the solutions of system (3.27) and system (3.28), we get

$$
D^{+} V(t) \leq \tilde{D}_{1}(t)+\tilde{D}_{2}(t)+2 A\left[\left|x_{2}(t)-x_{1}(t)\right|-\left|x_{2}(t-\tau)-x_{1}(t-\tau)\right|\right]
$$

where

$$
\begin{aligned}
& \tilde{D}_{1}(t)=\left\{\begin{array}{lr}
\frac{\dot{x}_{2}}{x_{2}}-\frac{\dot{x}_{1}}{x_{1}}=2 B\left[y_{2}(t)-y_{1}(t)\right]+\left[\varepsilon a-\frac{m_{1}}{x_{2}(t)}\right]\left[x_{2}(t)-x_{1}(t)\right], & x_{2}(t)>x_{1}(t), \\
\frac{\dot{x}_{1}}{x_{1}}-\frac{\dot{x}_{2}}{x_{2}}=-2 B\left[y_{2}(t)-y_{1}(t)\right]-\left[\varepsilon a-\frac{m_{1}}{x_{2}(t)}\right]\left[x_{2}(t)-x_{1}(t)\right], & x_{2}(t)<x_{1}(t), \\
\left|\frac{\dot{x}_{2}}{x_{2}}-\frac{\dot{x}_{1}}{x_{1}}\right|=2 B\left|y_{2}(t)-y_{1}(t)\right|, & x_{2}(t)=x_{1}(t),
\end{array}\right. \\
& \tilde{D}_{2}(t)=\left\{\begin{array}{lr}
\dot{y}_{2}-\dot{y}_{1}=-2 A\left[x_{2}(t-\tau)-x_{1}(t-\tau)\right]-\left[B\left(y_{2}(t)+y_{1}(t)\right)+m_{2}\right] e_{2}(t), & y_{2}(t)>y_{1}(t), \\
\dot{y}_{1}-\dot{y}_{2}=2 A\left[x_{2}(t-\tau)-x_{1}(t-\tau)\right]+\left[B\left(y_{2}(t)+y_{1}(t)\right)+m_{2}\right] e_{2}(t), & y_{2}(t)<y_{1}(t), \\
\left|\dot{y}_{2}-\dot{y}_{1}\right|=2 A\left|x_{2}(t-\tau)-x_{1}(t-\tau)\right|, & y_{2}(t)=y_{1}(t) .
\end{array}\right.
\end{aligned}
$$

There are the following three cases to consider for $\widetilde{D}_{1}(t)$. 
Journal of Applied Mathematics

(i) If $x_{2}(t)>x_{1}(t)$, then

$$
\begin{aligned}
\tilde{D}_{1}(t) & =2 B\left[y_{2}(t)-y_{1}(t)\right]+\left[\varepsilon a-\frac{m_{1}}{x_{2}(t)}\right]\left[x_{2}(t)-x_{1}(t)\right] \\
& \leq 2 B\left|y_{2}(t)-y_{1}(t)\right|+\left(\varepsilon a-m_{1}\right)\left|x_{2}(t)-x_{1}(t)\right| .
\end{aligned}
$$

(ii) If $x_{2}(t)<x_{1}(t)$, then

$$
\begin{aligned}
\tilde{D}_{1}(t) & =-2 B\left[y_{2}(t)-y_{1}(t)\right]-\left[\varepsilon a-\frac{m_{1}}{x_{2}(t)}\right]\left[x_{2}(t)-x_{1}(t)\right] \\
& \leq 2 B\left|y_{2}(t)-y_{1}(t)\right|+\left(\varepsilon a-m_{1}\right)\left|x_{2}(t)-x_{1}(t)\right| .
\end{aligned}
$$

(iii) If $x_{2}(t)=x_{1}(t)$, then

$$
\begin{aligned}
\widetilde{D}_{1}(t) & =2 B\left|y_{2}(t)-y_{1}(t)\right| \\
& =2 B\left|y_{2}(t)-y_{1}(t)\right|+\left(\varepsilon a-m_{1}\right)\left|x_{2}(t)-x_{1}(t)\right|
\end{aligned}
$$

From (i)-(iii), we have

$$
\widetilde{D}_{1}(t) \leq\left(\varepsilon a-m_{1}\right)\left|x_{2}(t)-x_{1}(t)\right|+2 B\left|y_{2}(t)-y_{1}(t)\right|, \quad t \geq t_{0} .
$$

Considering $\widetilde{D}_{2}(t)$ in the same way, we can obtain

$$
\tilde{D}_{2}(t) \leq 2 A\left|x_{2}(t-\tau)-x_{1}(t-\tau)\right|+\left(2 B-m_{2}\right)\left|y_{2}(t)-y_{1}(t)\right|, \quad t \geq t_{0} .
$$

Hence, we have

$$
\begin{aligned}
D^{+} V(t) & \leq \tilde{D}_{1}(t)+\tilde{D}_{2}(t)+2 A\left[\left|x_{2}(t)-x_{1}(t)\right|-\left|x_{2}(t-\tau)-x_{1}(t-\tau)\right|\right] \\
& \leq\left(2 A+\varepsilon a-m_{1}\right)\left|x_{2}(t)-x_{1}(t)\right|+\left(4 B-m_{2}\right)\left|y_{2}(t)-y_{1}(t)\right|, \quad t \geq t_{0} .
\end{aligned}
$$

So there exists $\alpha>0$ such that

$$
D^{+} V(t) \leq-\alpha\left(\left|x_{2}(t)-x_{1}(t)\right|+\left|y_{2}(t)-y_{1}(t)\right|\right), \quad t \geq t_{0} .
$$

Integrating on both sides of the above inequality leads to

$$
V(t)+\alpha \int_{t_{0}}^{t}\left(\left|x_{2}(s)-x_{1}(s)\right|+\left|y_{2}(s)-y_{1}(s)\right|\right) d s \leq V\left(t_{0}\right)<+\infty, \quad t \geq t_{0},
$$




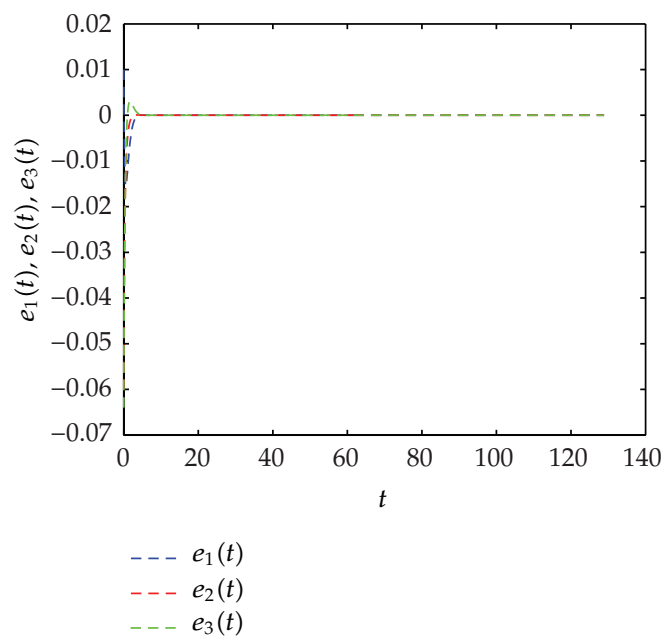

(a)

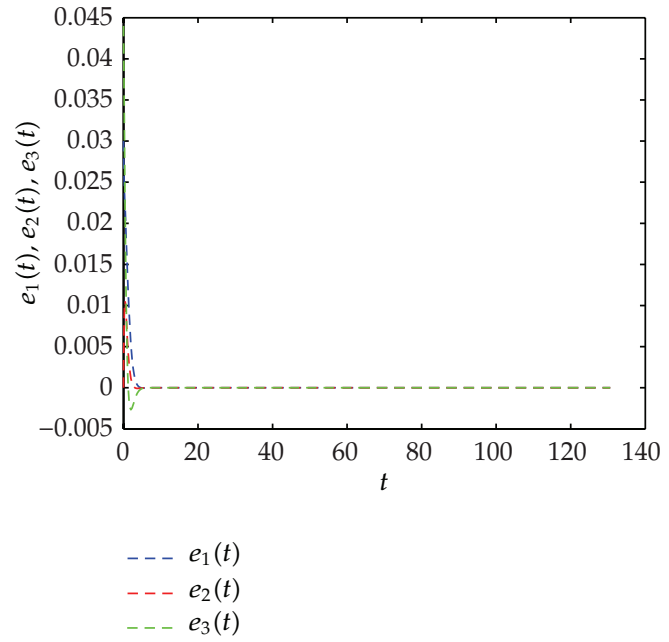

(b)

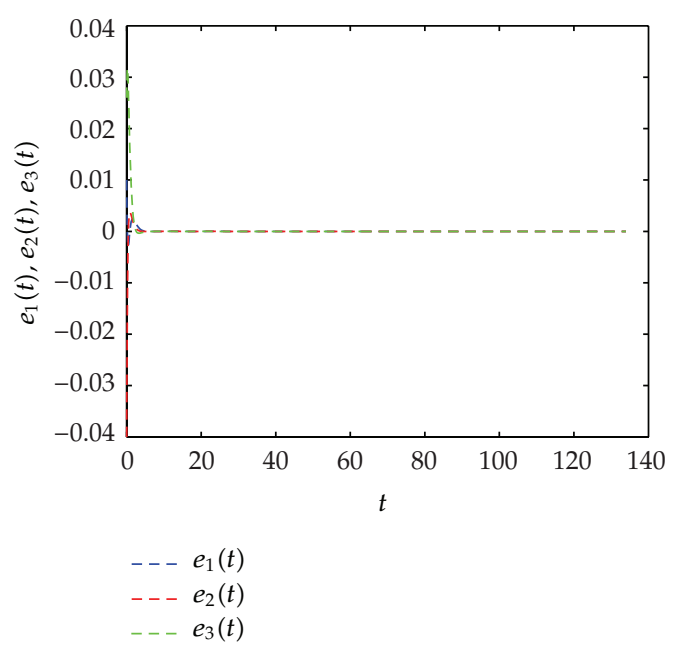

(c)

Figure 3: Synchronization errors $e_{1}(t), e_{2}(t)$, and $e_{3}(t)$ for the drive system (3.5) and response system (3.6). (a) $\tau=0.001, m_{1}=1.1761, m_{2}=3.31136, m_{3}=1.001, k_{1}=1, k_{2}=-3, k_{3}=-3$. (b) $\tau=0.003, m_{1}=1.1761$, $m_{2}=3.31136, m_{3}=1.001, k_{1}=-1, k_{2}=3, k_{3}=3$. (c) $\tau=0.01, m_{1}=1.1761, m_{2}=3.31136, m_{3}=1.001, k_{1}=1$, $k_{2}=-1, k_{3}=2$.

which implies

$$
\left|x_{2}(t)-x_{1}(t)\right|+\left|y_{2}(t)-y_{1}(t)\right| \in L^{1}[0,+\infty)
$$

From the boundedness of $x_{i}(t)$ and $y_{i}(t)(i=1,2)$, it follows that $x_{2}(t)-x_{1}(t), y_{2}(t)-y_{1}(t)$ and their derivatives remain bounded on $[0,+\infty)$. Hence, $\left|x_{2}(t)-x_{1}(t)\right|+\left|y_{2}(t)-y_{1}(t)\right|$ is uniformly continuous. By Lemma 3.1, it follows that

$$
\lim _{t \rightarrow+\infty}\left(\left|x_{2}(t)-x_{1}(t)\right|+\left|y_{2}(t)-y_{1}(t)\right|\right)=0 \text {. }
$$




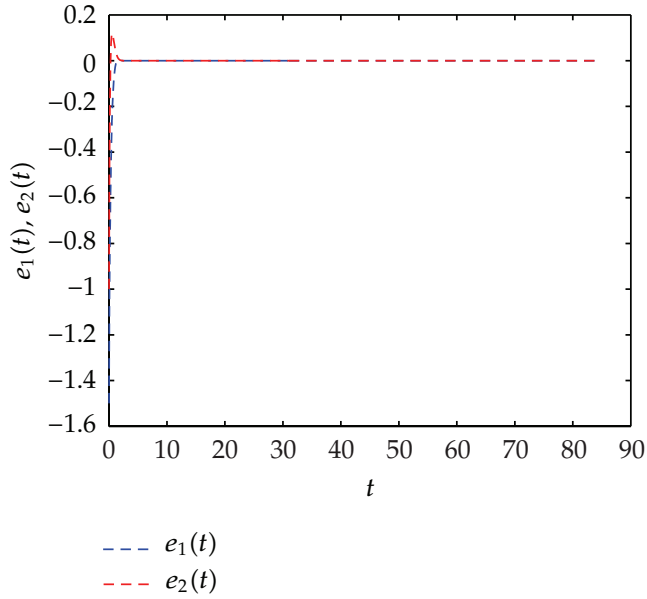

(a)

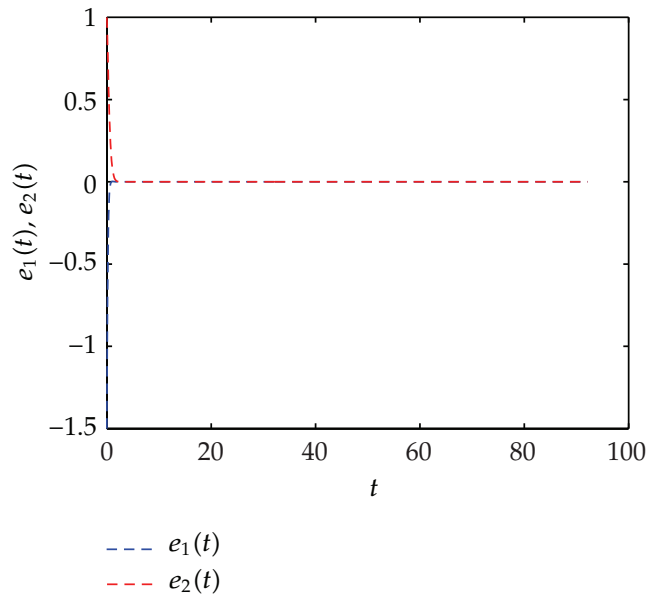

(b)

Figure 4: Synchronization errors $e_{1}(t), e_{2}(t)$ for the drive system (3.27) and response system (3.28). (a) $\tau=0.03, T=8, m_{1}=3.301, m_{2}=4.1$. (b) $\tau=0.03, T=10, m_{1}=3.231, m_{2}=4.01$.

Hence,

$$
\lim _{t \rightarrow+\infty}\left|x_{2}(t)-x_{1}(t)\right|=0, \quad \lim _{t \rightarrow+\infty}\left|y_{2}(t)-y_{1}(t)\right|=0
$$

This result implies that $e_{1}(t), e_{2}(t)$ converge to zero as time $t$ tends to infinity, that is, the drive system (3.27) and the response system (3.28) asymptotically synchronize. The proof is complete.

\section{Numerical Simulations}

In this section, simulation results are given below to illustrate the validity of the proposed method. Numerical simulations are carried out using MATLAB. DDE23 solver is adopted to solve delay differential equations in Section 3. follows:

The initial values of the drive and response systems in simulations are chosen as

(1) the initial values of systems (3.5) and (3.6): $x_{1}(s)=0.01, y_{1}(s)=-0.01, z_{1}(s)=$ -0.018 and $x_{2}(s)=0.02, y_{2}(s)=-0.03, z_{2}(s)=-0.01$, for all $s \in[-\tau, 0]$;

(2) the initial values of systems (3.27) and (3.28): (i) when $T=8, x_{1}(s)=0.85, y_{1}(s)=$ 0.5 and $x_{2}(s)=-0.65, y_{2}(s)=-0.5$, for all $s \in[-\tau, 0]$. (ii) when $T=10, x_{1}(s)=0.85$, $y_{1}(s)=-0.5$ and $x_{2}(s)=-0.65, y_{2}(s)=0.5$, for all $s \in[-\tau, 0]$.

The dynamics of synchronization errors for the drive and response systems are shown in Figures 3 and 4. It can be seen in these figures that the synchronization errors will rapidly converge to zero, that is to say, with the passage of time, by means of the linear control method, rank-one chaotic systems without and with delay are ultimately synchronous. 


\section{Conclusions}

In this paper, the presence of chaos in rank-one chaotic systems with delay are confirmed via a binary test (called 0-1 test) for chaos. The synchronization problems for rank-one chaotic systems are discussed. The linear delayed feedback control is used to achieve the synchronization between two rank-one chaotic systems without and with delay. The numerical simulations are in excellent agreement with the analytical results.

\section{Acknowledgments}

This research is supported by the National Natural Science Foundation of China (Grant nos. 10971085, 11061016). The author would like to thank Dr. Paul Matthews, University of Nottingham, for providing MATLAB codes used for computing the median $k$ of $k_{c}$ values. The author also would like to thank the referees for their valuable comments.

\section{References}

[1] L. M. Pecora and T. L. Carroll, "Synchronization in chaotic systems," Physical Review Letters, vol. 64, no. 8, pp. 821-824, 1990.

[2] R. Mainieri and J. Rehacek, "Projective synchronization in three-dimensional chaotic systems," Physical Review Letters, vol. 82, no. 15, pp. 3042-3045, 1999.

[3] J. Yan and C. Li, "Generalized projective synchronization of a unified chaotic system," Chaos, Solitons and Fractals, vol. 26, no. 4, pp. 1119-1124, 2005.

[4] G. Wen and D. Xu, "Nonlinear observer control for full-state projective synchronization in chaotic continuous-time systems," Chaos, Solitons and Fractals, vol. 26, no. 1, pp. 71-77, 2005.

[5] Y. W. Wang and Z. H. Guan, "Generalized synchronization of continuous chaotic system," Chaos, Solitons and Fractals, vol. 27, no. 1, pp. 97-101, 2006.

[6] D. Xu and C. Y. Chee, "Controlling the ultimate state of projective synchronization in chaotic systems of arbitrary dimension," Physical Review E, vol. 66, no. 4, Article ID 046218, 5 pages, 2002.

[7] D. Xu, "Control of projective synchronization in chaotic systems," Physical Review E, vol. 63, no. 2, Article ID 027201, 4 pages, 2001.

[8] Z. Li and D. Xu, "Stability criterion for projective synchronization in three-dimensional chaotic systems," Physics Letters, Section A: General, Atomic and Solid State Physics, vol. 282, no. 3, pp. 175-179, 2001.

[9] C. Y. Chee and D. Xu, "Secure digital communication using controlled projective synchronisation of chaos," Chaos, Solitons and Fractals, vol. 23, no. 3, pp. 1063-1070, 2005.

[10] G. Álvarez, S. Li, F. Montoya, G. Pastor, and M. Romera, "Breaking projective chaos synchronization secure communication using filtering and generalized synchronization," Chaos, Solitons and Fractals, vol. 24, no. 3, pp. 775-783, 2005.

[11] J. H. Park, "Adaptive controller design for modified projective synchronization of Genesio-Tesi chaotic system with uncertain parameters," Chaos, Solitons and Fractals, vol. 34, no. 4, pp. 1154-1159, 2007.

[12] G. H. Li, "Generalized projective synchronization of two chaotic systems by using active control," Chaos, Solitons and Fractals, vol. 30, no. 1, pp. 77-82, 2006.

[13] W. He and J. Cao, "Generalized synchronization of chaotic systems: An auxiliary system approach via matrix measure," Chaos, vol. 19, no. 1, Article ID 013118, 2009.

[14] J. Cao, D. W. C. Ho, and Y. Yang, "Projective synchronization of a class of delayed chaotic systems via impulsive control," Physics Letters, Section A: General, Atomic and Solid State Physics, vol. 373, no. 35, pp. 3128-3133, 2009.

[15] W. Xia and J. Cao, "Pinning synchronization of delayed dynamical networks via periodically intermittent control," Chaos, vol. 19, no. 1, Article ID 013120, 2009.

[16] J. Cao, Z. Wang, and Y. Sun, "Synchronization in an array of linearly stochastically coupled networks with time delays," Physica A: Statistical Mechanics and its Applications, vol. 385, no. 2, pp. 718-728, 2007.

[17] Q. Wang and L. S. Young, "Strange attractors with one direction of instability," Communications in Mathematical Physics, vol. 218, no. 1, pp. 1-97, 2001. 
[18] Q. Wang and L. S. Young, "From invariant curves to strange attractors," Communications in Mathematical Physics, vol. 225, no. 2, pp. 275-304, 2002.

[19] Q. Wang and L. S. Young, "Strange Attractors in Periodically-Kicked Limit Cycles and Hopf Bifurcations," Communications in Mathematical Physics, vol. 240, no. 3, pp. 509-529, 2003.

[20] Q. Wang and A. Oksasoglu, "Strange attractors in periodically kicked Chua's circuit," International Journal of Bifurcation and Chaos in Applied Sciences and Engineering, vol. 15, no. 1, pp. 83-98, 2005.

[21] F. Chen and M. Han, "Rank one chaos in a class of planar systems with heteroclinic cycle," Chaos, vol. 19, no. 4, Article ID 043122, 2009.

[22] G. A. Gottwald and I. Melbourne, "A new test for chaos in deterministic systems," Proceedings of the Royal Society A: Mathematical, Physical and Engineering Sciences, vol. 460, no. 2042, pp. 603-611, 2004.

[23] G. A. Gottwald and I. Melbourne, “Testing for chaos in deterministic systems with noise," Physica D: Nonlinear Phenomena, vol. 212, no. 1-2, pp. 100-110, 2005.

[24] G. A. Gottwald and I. Melbourne, "On the implementation of the $0-1$ test for chaos," SIAM Journal on Applied Dynamical Systems, vol. 8, no. 1, pp. 129-145, 2009.

[25] K. Gopalsamy, Stability and Oscillations in Delay Differential Equations of Population Dynamics, vol. 74 of Mathematics and its Applications, Kluwer Academic Publishers Group, Dordrecht, The Netherlands, 1992. 


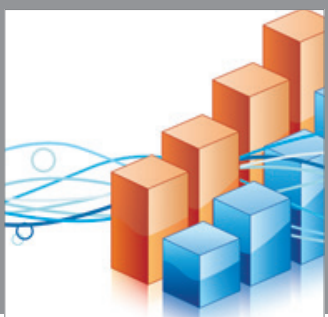

Advances in

Operations Research

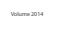

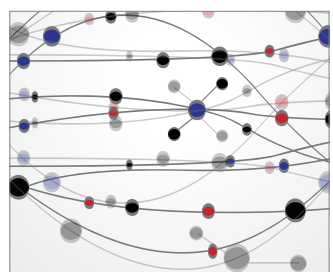

\section{The Scientific} World Journal
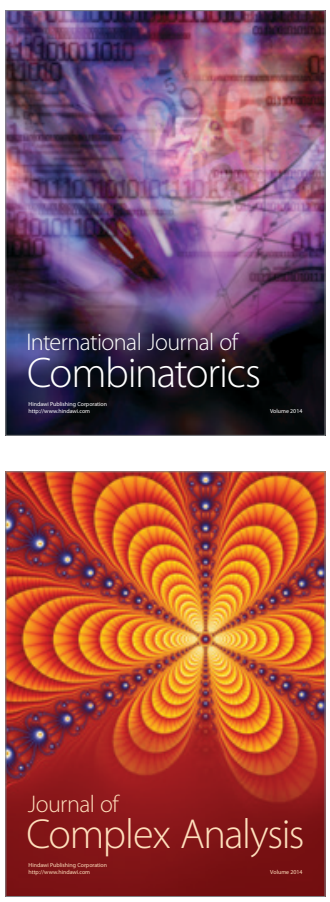

International Journal of

Mathematics and

Mathematical

Sciences
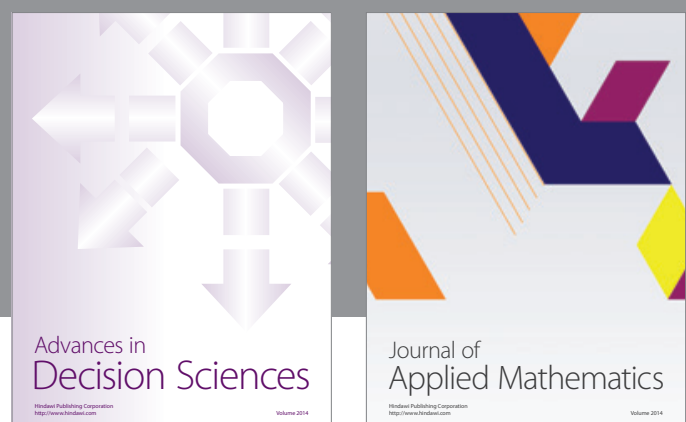

Journal of

Applied Mathematics
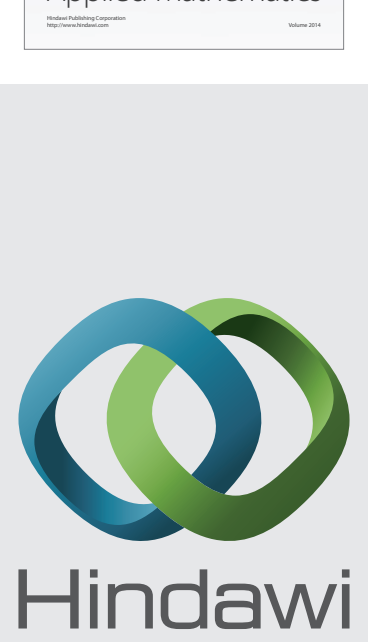

Submit your manuscripts at http://www.hindawi.com
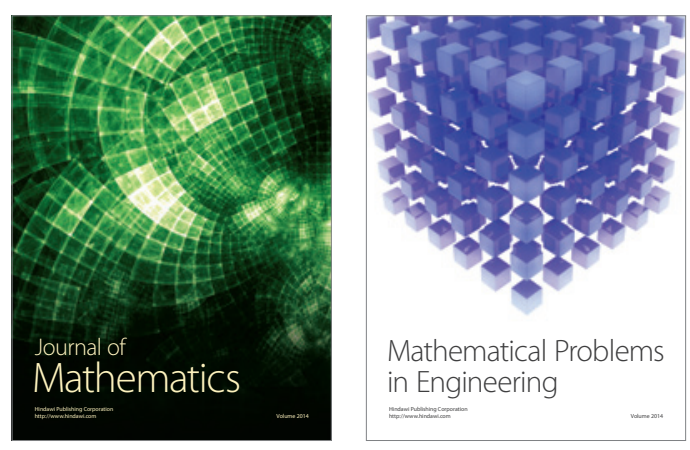

Mathematical Problems in Engineering
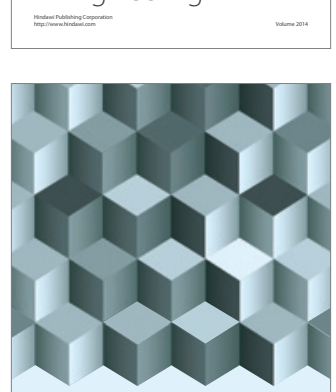

Journal of

Function Spaces
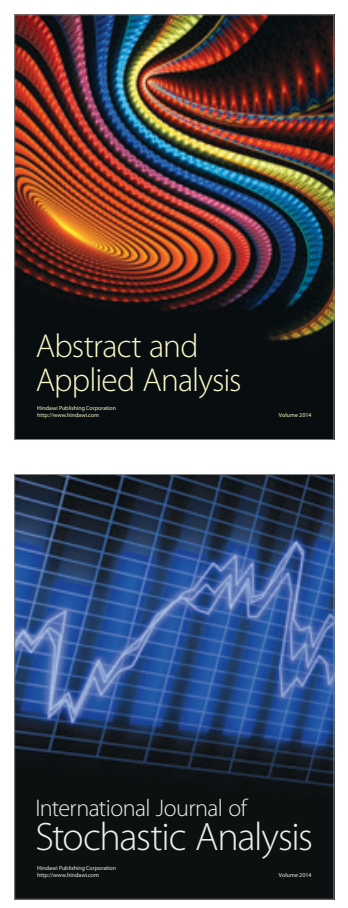

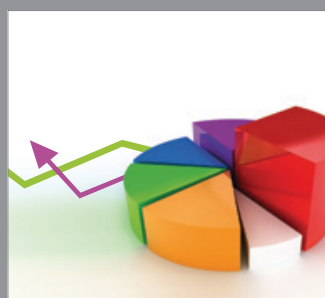

ournal of

Probability and Statistics

Promensencen
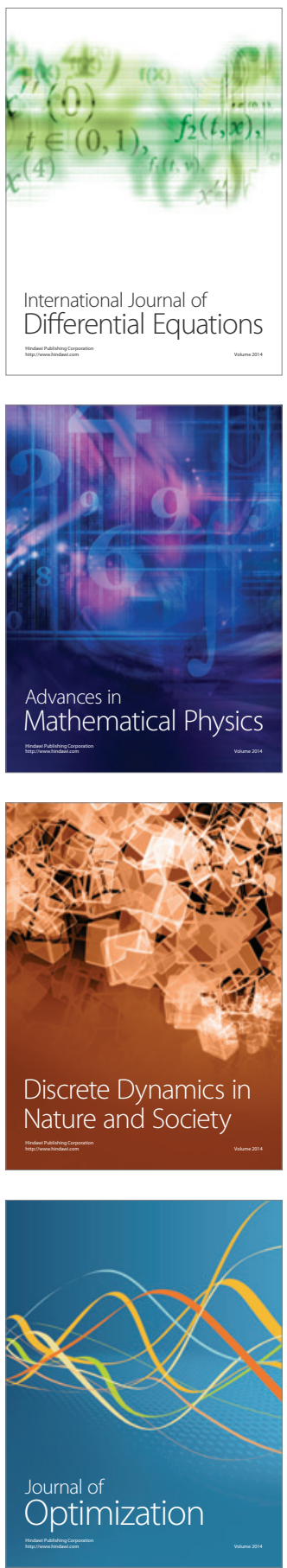